\title{
Indicadores económicos de las distribuidoras de maíz y su relación con la economía del cantón la Maná.
}

Economic indicators of the distributors of maize and its relationship with the economy of the canton the Mana.

Brenda Elizabeth Oña Sinchiguano. ${ }^{1}$, Héctor Arnulfo Chacha Armas. ${ }^{2}$, Amable Bienvenido Bravo. ${ }^{3}$, Carrasco Ruano Tatiana. ${ }^{4} \&$ Elsa Marlene Tixilema Chiliquinga. ${ }^{5}$

\begin{abstract}
.
DOI: https://doi.org/10.33262/cienciadigital.v2i3.136

The purpose of this research work is to determine the economic position of corn distributors and their relationship with the economy of La Maná county, Cotopaxi province, 2011. The problem is the lack of analysis and monitoring of economic information, to determine whether or not there are benefits in maize distributors, which results in inadequate decision making when determining prices, costs and expenses, all these factors create uncertainty in marketers and their workers about the continuity of their operations. To carry out the analysis and interpretation of the results of the research, the technological support was used in the preparation of mathematical tables and statistical graphs, which allows arriving at conclusions about the profitability of corn commercialization.

The profitability study is presented to the financial statements of corn trading companies: La Union, Erick and Kelvin, based on the analysis and interpretation of the financial statements of the three distributors dedicated to the same economic activity.
\end{abstract}

Keywords: Indicadores económicos, economía. VAN, TIR

${ }^{1}$ Universidad Técnica de Cotopaxi, Cotopaxi, Ecuador, brenda.ona@utc.edu.ec

${ }^{2}$ Universidad Técnica de Cotopaxi, Cotopaxi, Ecuador, hector.chacha@utc.edu.ec

${ }^{3}$ Universidad Técnica de Cotopaxi, Cotopaxi, Ecuador, amable.bravo@utc.edu.ec

${ }^{4}$ Visionario Digital, Editora Ambato, Ecuador. tatianacarrasco@cienciadigital.org

${ }^{5}$ Universidad Técnica de Cotopaxi, Cotopaxi, Ecuador, elsa.tixilema@utc.edu.ec 


\section{Resumen.}

El presente trabajo de investigación tiene como finalidad determinar la rentabilidad de las distribuidoras de maíz y su relación con la economía del cantón La Maná, provincia de Cotopaxi, año 2011. El problema es la falta de un análisis y seguimiento de la información financiera, para determinar si existe o no rentabilidad en las distribuidoras de maíz, que tiene como resultado una inadecuada toma decisiones al momento de determinar precios, costos y gastos, todos estos factores crean incertidumbre en las comercializadoras y en sus trabajadores acerca de la continuidad de sus operaciones. Para la realización del análisis e interpretación de resultados de la investigación se utilizó el soporte tecnológico en la elaboración de cuadros matemáticos y gráficos estadísticos, lo que permite arribar a conclusiones acerca de la rentabilidad de la comercialización de maíz.

Se presenta el estudio de rentabilidad a los estados financieros de las empresas comercializadoras de maíz: La Unión, Erick y Kelvin, a partir del análisis e interpretación de los estados financieros de las tres distribuidoras dedicadas a la misma actividad económica.

Palabras claves: Indicadores económicos, economía. VAN, TIR

\section{Introducción.}

La presente investigación permitió determinar las tasas de rentabilidad que generan las distribuidoras de maíz y su relación con la economía del cantón

La Maná , el estudio bibliográfico se centra en la metodología del análisis financiero con la aplicación del VAN, TIR que nos ayuda a establecer la tasa de rentabilidad del negocio.

Fue necesaria la recopilación de información financiera y la utilización de las técnicas matemáticas para obtener resultados.

Se determinó además que las empresas trabajan empíricamente sin un plan estratégico o presupuestos de ventas y gastos por lo cual no poseen ninguna información histórica en sus registros financieros, más que los exigidos por los organismos de control.

Esta información al no ser sometida a un análisis financiero o enfrentado con un plan de negocio no proporciona ningún tipo de ayuda a sus inversionistas, mismos que trabajan siempre con la incertidumbre si sus operaciones son realmente rentables y si podrán ser sustentables sus actividades a un futuro. 


\section{Metodología.}

En la investigación se utilizó métodos de análisis de indicadores económicos de la información recopilada de las comercializadoras de maíz, en el cantón La Maná. El obtener los márgenes de rentabilidad permitió conocer las ganancias de los inversionistas para generar resultados de la investigación y obtener de manera específica índices financieros que permitan un análisis objetivo de la situación financiera y solidez de los negocios.

Se aplicaron los principales indicadores económicos para el análisis de la información recopilada de las comercializadoras de maíz.

Indicadores Económicos

- Indicadores de Riqueza (cantidad de circulante)

$$
\begin{aligned}
& >\mathrm{VAN} \\
& >\mathrm{VAE}
\end{aligned}
$$

- Indicadores de Rentabilidad (velocidad en la generación de riqueza)

$>$ TIR

$>$ TIR CORREGIDA

$>\mathrm{VAN} / \mathrm{INV}$.

- Indicadores Misceláneas

$>$ Razón Beneficio Costo

$>$ Periodo de recuperación (payback)

VAN (Valor Actual Neto).- Posibilita de una manera simple y exacta el valor neto del proyecto para el horizonte del planeamiento. Para la aplicación de fórmulas y la tasa de descuento que reflejan el costo de oportunidad del capital para aceptar, postergar o desechar el proyecto según el resultado.

Es muy importante el momento en el que se perciben los beneficios. A medida que es mayor la tasa de descuento, menos importantes son los costos e ingresos que se generan en el futuro y de mayor importancia los costos cercanos al inicio del proyecto.

Un proyecto es rentable para el inversionista si el VAN es mayor que CERO.

- VAN $>0$ Proyecto rentable

- VAN $<0$ Proyecto no rentable

- $\mathrm{VAN}=0$ Proyecto indiferente 
Formula

$$
V A N=-I+\sum_{n=1}^{N} \frac{Q_{n}}{(1+r)^{n}}
$$

van: valor actual neto.

qn: representa los flujos de caja en cada periodo $\mathrm{n}$.

i: inversión inicial.

n: número de periodos considerados.

r: tasa de descuento

Tir (tasa interna de retorno).- conocida también como tasa interno de rendimiento, es un instrumento o medida usada como indicador al momento de revisar la eficacia de una inversión. La tir sirve para identificar claramente el tiempo en el que recuperaremos el capital asignado a una inversión. Para su cálculo se requiere proyectar los gastos e ingresos en periodos regulares.

Formula

$$
T I R=\sum_{T=0}^{n} \frac{F n}{(1+i)^{n}}=0
$$

Fn: flujo del efectivo anual.

n: vida útil del proyecto.

\section{Flujos de caja}

Tabla 1: Flujo de Caja de Distribuidora La Unión.

\begin{tabular}{|l|r|r|r|r|r|r|}
\hline \multirow{2}{*}{ CUENTAS } & \multirow{2}{*}{$\begin{array}{l}\text { INVERSION } \\
\text { INICIAL }\end{array}$} & $\mathbf{1}$ & $\mathbf{2}$ & \multicolumn{1}{c|}{$\mathbf{3}$} & $\mathbf{4}$ & $\mathbf{5}$ \\
\cline { 5 - 7 } & & & & & & \\
& & & & & \\
\hline A. INGRESOS OPERACIONALES & & $\$ 234.000,00$ & $\$ 246.659,40$ & $\$ 260.003,67$ & $\$ 274.069,87$ & $\$ 288.897,05$ \\
\hline Clientes & & $\$ 12.435,99$ & $\$ 13.108,78$ & $\$ 13.817,96$ & $\$ 14.565,51$ & $\$ 15.353,51$ \\
\hline Total de ingresos operacionales & & $\mathbf{\$ 2 4 6 . 4 3 5 , 9 9}$ & $\mathbf{\$ 2 5 9 . 7 6 8 , 1 8}$ & $\mathbf{\$ 2 7 3 . 8 2 1 , 6 4}$ & $\mathbf{\$ 2 8 8 . 6 3 5 , 3 9}$ & $\mathbf{\$ 3 0 4 . 2 5 0 , 5 6}$ \\
\hline B. EGRESOS OPERACIONALES & & & & & & \\
\hline Costos Ventas & & $\$ 190.372,00$ & $\$ 200.671,13$ & $\$ 211.527,43$ & $\$ 222.971,07$ & $\$ 235.033,80$ \\
\hline Gastos Comercialización & & $\$ 8.000,00$ & $\$ 8.432,80$ & $\$ 8.889,01$ & $\$ 9.369,91$ & $\$ 9.876,82$ \\
\hline Gastos de administración & & $\$ 14.533,33$ & $\$ 15.319,59$ & $\$ 16.148,38$ & $\$ 17.022,00$ & $\$ 17.942,89$ \\
\hline
\end{tabular}


ISSN: 2602-8085

www.cienciadigital.org

Vol. 2, N³, p. 50-60, Julio - Septiembre, 2018

\begin{tabular}{|c|c|c|c|c|c|c|}
\hline Gastos de venta & & $\$ 4.550,00$ & $\$ 4.796,16$ & $\$ 5.055,63$ & $\$ 5.329,14$ & $\$ 5.617,44$ \\
\hline Total & & $\$ 217.455,33$ & $\$ 20.115,74$ & $\$ 21.204,00$ & $\$ 22.351,14$ & $\$ 23.560,34$ \\
\hline C. FLUJO OPERACIONAL (A-B) & & $\$ 28.980,66$ & $\$ 239.652,43$ & $\$ 252.617,63$ & $\$ 266.284,25$ & $\$ 280.690,22$ \\
\hline \multicolumn{7}{|l|}{$\begin{array}{l}\text { D. INGRESOS NO } \\
\text { OPERACIONALES }\end{array}$} \\
\hline \multicolumn{7}{|l|}{ Valor residual de activos } \\
\hline Valor residual capital de trabajo & & $\$ 0,00$ & $\$ 21.753,20$ & $\$ 35.887,94$ & $\$ 63.745,27$ & $\$ 119.025,47$ \\
\hline \multicolumn{7}{|l|}{ Total ingresos no operacionales } \\
\hline \multicolumn{7}{|l|}{$\begin{array}{l}\text { E. EGRESOS NO } \\
\text { OPERACIONALES }\end{array}$} \\
\hline Vehículo & $\$ 17.000,00$ & & & & & \\
\hline Edificios & $\$ 25.000,00$ & & & & & \\
\hline Equipos de oficina & $\$ 900,00$ & & & & & \\
\hline Muebles de oficina & $\$ 2.000,00$ & & & & & \\
\hline Capital de trabajo & $\$ 21.753,20$ & & & & & \\
\hline Pago de impuesto a la renta & & $\$ 1.052,98$ & $\$ 1.109,95$ & $\$ 1.169,99$ & $\$ 1.233,29$ & $\$ 1.300,01$ \\
\hline Pago créditos & & $\$ 2.737,44$ & $\$ 2.885,54$ & $\$ 3.041,64$ & $\$ 3.206,20$ & $\$ 3.379,65$ \\
\hline Pago $15 \%$ a trabajadores & & $\$ 3.437,03$ & $\$ 3.622,98$ & $\$ 3.818,98$ & $\$ 4.025,59$ & $\$ 4.243,37$ \\
\hline Total de egresos no operacionales & $\$ 66.653,20$ & $\$ 7.227,45$ & $\$ 7.618,46$ & $\$ 27.857,33$ & $\$ 8.465,07$ & $\$ 8.923,04$ \\
\hline F. FLUJO NO OPERACIONAL & $-\$ 66.653,20$ & $-\$ 7.227,45$ & $\$ 14.134,74$ & $\$ 8.030,62$ & $\$ 55.280,20$ & $-\$ 7.623,02$ \\
\hline $\begin{array}{l}\text { G. FLUJO NETO GENERADOR } \\
(\mathrm{C}+\mathrm{F})\end{array}$ & $-\$ 66.653,20$ & $\$ 21.753,20$ & $\$ 35.887,94$ & $\$ 63.745,27$ & $\$ 119.025,47$ & $\$ 127.948,50$ \\
\hline TASA DE DESCUENTO & $20 \%$ & & & & & \\
\hline VAN & $\$ 55.544,33$ & & & & & \\
\hline TIR & $65 \%$ & & & & & \\
\hline
\end{tabular}

Elaborado por: Grupo de Investigación.

Tabla 2: Flujo de Caja de Distribuidora Erick

\begin{tabular}{|l|r|r|r|r|r|r|}
\hline \multirow{2}{*}{ CUENTAS } & \multirow{2}{*}{$\begin{array}{l}\text { INVERSION } \\
\text { INICIAL }\end{array}$} & \multicolumn{5}{c|}{ ANOS } \\
\cline { 5 - 7 } & & $\mathbf{1}$ & $\mathbf{2}$ & $\mathbf{3}$ & $\mathbf{4}$ & $\mathbf{5}$ \\
\hline A. INGRESOS OPERACIONALES & & & & & & \\
\hline Ventas Maíz & & $\$ 114.120,00$ & $\$ 120.293,89$ & $\$ 126.801,79$ & $\$ 133.661,77$ & $\$ 140.892,87$ \\
\hline Clientes & & $\$ 8.167,50$ & $\$ 8.609,36$ & $\$ 9.075,13$ & $\$ 9.566,09$ & $\$ 10.083,62$ \\
\hline Total de ingresos operacionales & & $\$ \mathbf{1 2 2 . 2 8 7 , 5 0}$ & $\mathbf{\$ 1 2 8 . 9 0 3 , 2 5}$ & $\mathbf{\$ 1 3 5 . 8 7 6 , 9 2}$ & $\mathbf{\$ 1 4 3 . 2 2 7 , 8 6}$ & $\mathbf{\$ 1 5 0 . 9 7 6 , 4 9}$ \\
\hline B. EGRESOS OPERACIONALES & & & & & & \\
\hline Costos Ventas & & $\$ 83.024,00$ & $\$ 87.515,60$ & $\$ 92.250,19$ & $\$ 97.240,93$ & $\$ 102.501,66$ \\
\hline Gastos de comercialización & & $\$ 36.000,00$ & $\$ 37.947,60$ & $\$ 40.000,57$ & $\$ 42.164,60$ & $\$ 44.445,70$ \\
\hline Gastos de administración & & $\$ 14.738,95$ & $\$ 15.536,32$ & $\$ 16.376,84$ & $\$ 17.262,82$ & $\$ 18.196,74$ \\
\hline Gastos de venta & & $\$ 3.882,50$ & $\$ 4.092,54$ & $\$ 4.313,95$ & $\$ 4.547,33$ & $\$ 4.793,35$ \\
\hline
\end{tabular}




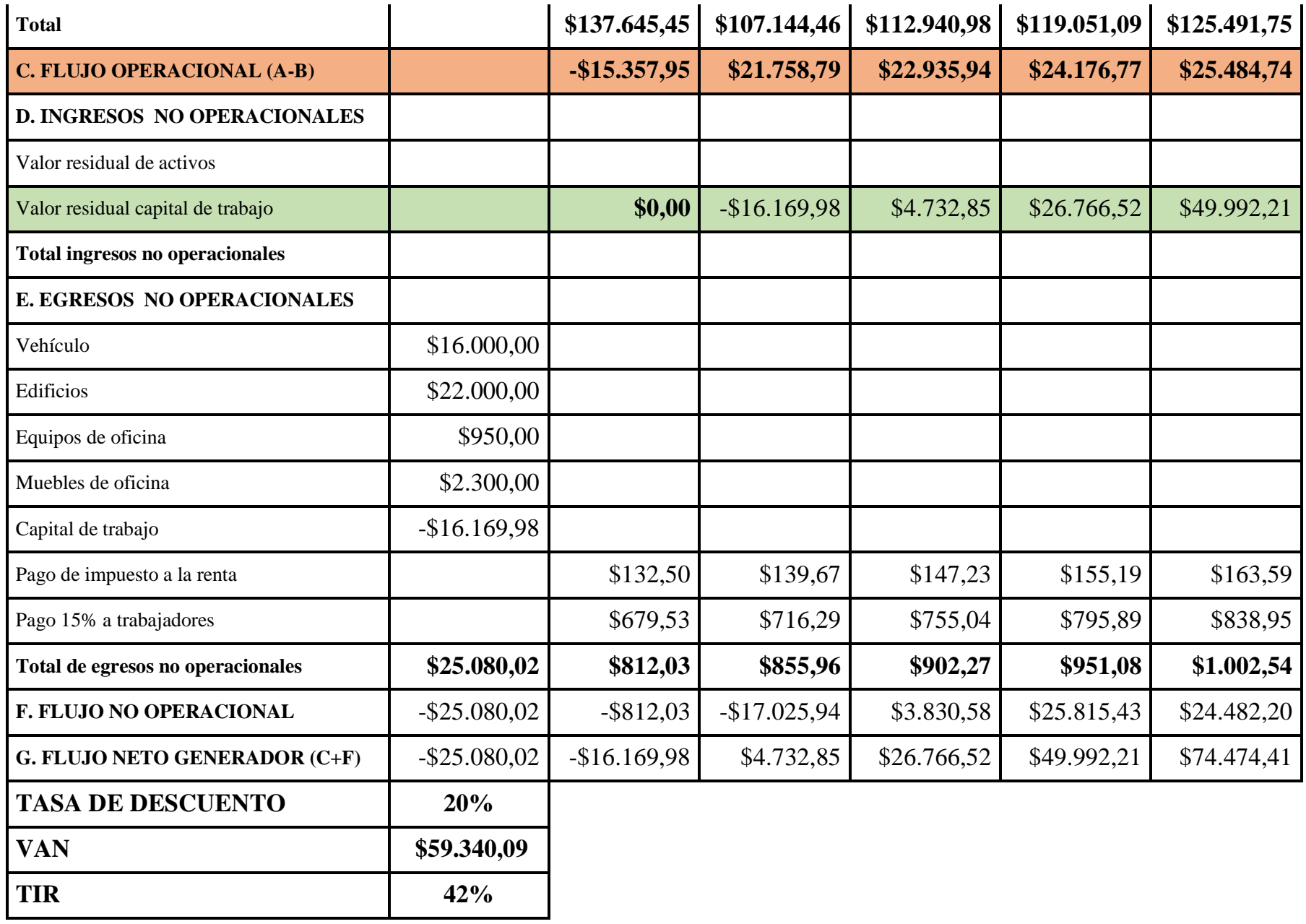

Elaborado por: Grupo de Investigación.

Tabla 3: Flujo de Caja de Distribuidora Kelvin

\begin{tabular}{|l|l|r|r|r|r|r|}
\hline \multirow{2}{*}{ CUENTAS } & \multirow{2}{*}{$\begin{array}{l}\text { INVERSION } \\
\text { INICIAL }\end{array}$} & \multicolumn{5}{c|}{ ANOS } \\
\cline { 5 - 7 } & & $\mathbf{1}$ & $\mathbf{2}$ & $\mathbf{3}$ & $\mathbf{4}$ & $\mathbf{5}$ \\
\hline A. INGRESOS OPERACIONALES & & & & & & \\
\hline ventas de maíz & & $\$ 93.600,00$ & $\$ 98.663,76$ & $\$ 104.001,47$ & $\$ 109.627,95$ & $\$ 115.558,82$ \\
\hline Clientes & & $\$ 4.455,00$ & $\$ 4.696,02$ & $\$ 4.950,07$ & $\$ 5.217,87$ & $\$ 5.500,16$ \\
\hline Total de ingresos operacionales & & $\$ 98.055,00$ & $\$ 103.359,78$ & $\$ 108.951,54$ & $\$ 114.845,82$ & $\$ 121.058,98$ \\
\hline B. EGRESOS OPERACIONALES & & & & & & \\
\hline Costos de Venta & & $\$ 64.964,00$ & $\$ 68.478,55$ & $\$ 72.183,24$ & $\$ 76.088,36$ & $\$ 80.204,74$ \\
\hline Gasto de comercialización & & $\$ 30.000,00$ & $\$ 31.623,00$ & $\$ 33.333,80$ & $\$ 35.137,16$ & $\$ 37.038,08$ \\
\hline Gastos de administración & & $\$ 9.528,81$ & $\$ 10.044,32$ & $\$ 10.587,72$ & $\$ 11.164,75$ & $\$ 11.768,76$ \\
\hline Gastos de venta & & $\$ 6.645,00$ & $\$ 7.004,49$ & $\$ 7.383,44$ & $\$ 7.782,88$ & $\$ 8.203,94$ \\
\hline Total & & $\mathbf{\$ 1 1 1 . 1 3 7 , 8 1}$ & $\mathbf{\$ 8 5 . 5 2 7 , 3 7}$ & $\mathbf{\$ 9 0 . 1 5 4 , 4 0}$ & $\mathbf{\$ 9 5 . 0 3 5 , 9 9}$ & $\mathbf{\$ 1 0 0 . 1 7 7 , 4 3}$ \\
\hline
\end{tabular}




\begin{tabular}{|c|c|c|c|c|c|c|}
\hline C. FLUJO OPERACIONAL (A-B) & & $-\$ 13.082,81$ & $\$ 17.832,41$ & $\$ 18.797,14$ & $\$ 19.809,83$ & $\$ 20.881,54$ \\
\hline \multicolumn{7}{|l|}{ D. INGRESOS NO OPERACIONALES } \\
\hline \multicolumn{7}{|l|}{ Valor residual de activos } \\
\hline Valor residual capital de trabajo & & $\$ 0,00$ & $-\$ 15.021,28$ & $\$ 767,78$ & $\$ 17.411,04$ & $\$ 34.950,45$ \\
\hline Total ingresos no operacionales & & & $-\$ 15.021,28$ & $\$ 767,78$ & $\$ 17.411,04$ & $\$ 34.950,45$ \\
\hline \multicolumn{7}{|l|}{ E. EGRESOS NO OPERACIONALES } \\
\hline Edificio & $\$ 22.000,00$ & & & & & \\
\hline Escritorio & $\$ 560,00$ & & & & & \\
\hline Tableros & $\$ 450,00$ & & & & & \\
\hline Vehículo & $\$ 12.000,00$ & & & & & \\
\hline Capital de trabajo & $-\$ 15.021,28$ & & & & & \\
\hline Pago de impuesto a la renta & & $\$ 69,14$ & $\$ 72,88$ & $\$ 76,83$ & $\$ 80,98$ & $\$ 85,36$ \\
\hline Pago $15 \%$ a trabajadores & & $\$ 1.869,33$ & $\$ 1.970,46$ & $\$ 2.077,06$ & $\$ 2.189,43$ & $\$ 2.307,88$ \\
\hline Total de egresos no operacionales & $\$ 19.988,72$ & $\$ 1.938,47$ & $\$ 2.043,34$ & $\$ 2.153,89$ & $\$ 2.270,41$ & $\$ 2.393,24$ \\
\hline F. FLUJO NO OPERACIONAL & $-\$ 19.988,72$ & $-\$ 1.938,47$ & $-\$ 17.064,63$ & $-\$ 1.386,11$ & $\$ 15.140,62$ & $\$ 32.557,21$ \\
\hline G. FLUJO NETO GENERADOR (C+F) & $-\$ 19.988,72$ & $-\$ 15.021,28$ & $\$ 767,78$ & $\$ 17.411,04$ & $\$ 34.950,45$ & $\$ 53.438,76$ \\
\hline TASA DE DESCUENTO & $20 \%$ & & & & & \\
\hline VAN & $\$ 36.422,08$ & & & & & \\
\hline TIR & $34 \%$ & & & & & \\
\hline
\end{tabular}

Elaborado por: Grupo de Investigación.

El análisis de los índices económicos muestran un VAN positivo en cada una de las distribuidoras lo que hace viable cada uno de los negocios que están funcionando. La tasa de descuento aplicada es del $20 \%$ que sería la tasa mínima de retorno que esperan los inversionistas y la TIR en cada uno de los negocios es muy superior a la mínima esperada.

\section{Impacto en el cantón la Maná.}

El cantón La Maná tiene 40.655 habitantes según el VII Censo de Población y VI de Vivienda, realizado por el Instituto Ecuatoriano de Estadísticas y Censos (INEC) en noviembre del 2010.

La Población Económicamente Activa (PEA) del cantón La Maná suma 21.365 habitantes de los cuales solo el 46,91\% se encuentran empleados, mientras que el desempleo es del $7,8 \%$, el resto de habitantes son subempleados dedicados principalmente a tareas de comercio informal.

La población del cantón La Maná empleada en la distribución al por mayor de maíz es de 42 personas, mientras que el comercio minorista de maíz suma alrededor de 108 
empleados, es decir, que 150 habitantes de la localidad están empleados en empresas dedicadas a la distribución de maíz

A ellos se suman alrededor de 600 personas que trabajan en distribuidoras que compran maíz y lo comercializan al por menor, en tiendas y comisariatos, lo que incrementa el número de personas empleadas en las actividades de distribución de maíz a 750 habitantes, que representa el 3,51\% de la PEA y el 7,48\% de la población con empleo.

Esto significa que si la distribución de maíz desaparece del mercado lamanence, por lo menos 8 de cada 100 trabajadores quedarán en el desempleo, aumentando la tasa de desempleo en el 7,48\%, en cambio sí se incrementan las empresas distribuidoras de maíz en la localidad, podrían reducir en un porcentaje mayor al $2 \%$ la tasa de desempleo, lo que pone de manifiesto la importancia de la actividad en la economía del cantón La Maná.

Cada trabajador que labora en la distribución de maíz tiene un sueldo promedio de $\$ 650,00$ aproximadamente, donde los empleados administrativos tienen niveles promedios de sueldos de $\$ 870,00$ y los operadores devengan sueldos promedios de $\$ 350,00$, mientras que los vendedores comisionistas pueden ganar hasta $\$ 680,00$ en promedio.

La actividad de la distribución de maíz, representa alrededor del 2,2\% de los ingresos de todas las actividades productivas en el cantón La Maná, representando una importante fuente de ingresos para el trabajador lamanence.

En lo que corresponde a la relación entre la distribución de maíz en el cantón La Maná y su influencia en la economía ecuatoriana, se ha evidenciado que esta localidad participa con el 2,69\% de la distribución de maíz a nivel nacional, es decir, que de cada 100 sacos de maíz que se distribuyen en el Ecuador, 3 son distribuidos por algún establecimiento lamanence.

De acuerdo a la información proporcionada por el Banco Central, la distribución nacional de maíz representa el 3\% del PIB agrícola a nivel nacional, que a su vez representa el $15,49 \%$ del PIB de las ramas no petroleras, mientras que también la distribución de maíz participa con el 22,5\% del PIB.

Esto quiere decir, que de cada $\$ 100,00$ generados por todas las empresas que realizan actividades agrícolas, $\$ 3,00$ lo generan los establecimientos dedicados a la distribución de maíz

Por tanto, la actividad que generan las empresas dedicadas a la distribución de maíz podría afectar sensiblemente a la economía nacional, si es que desaparecen del mercado nacional, generando un incremento del desempleo y menores cargas impositivas para el empleado a trabajadores de la Maná. 


\section{Conclusiones.}

- La aplicación de los índices económicos del VAN y el TIR muestran VAN positivos, si el VAN es mayor de CERO es un indicador de rentabilidad del negocio ya que supera una tasa de descuento impuesta como mínima para retorno de los inversionistas. De igual manera la TIR es muy superior a la tasa del 20\% mínima para el cálculo de los dos índices.

- Se ha demostrado que la rentabilidad de la comercialización del maíz es amplia, las tres distribuidoras de estudio presenta un índice económico que los ubica como distribuidoras sólidas dentro de este mercado, lo que influye grandemente en la economía del cantón La Mana, como generadoras de empleo y de comercio en el mismo.

\section{Referencias bibliográficas.}

(Sánchez, I. R (2003) Enfoque económico social de evaluación de proyectos de inversión. Tesis doctoral en Ciencias Económica, CDICT, Universidad de la Habana).

(Sánchez, I. R. (2002) "Tópico especiales sobre evaluación de proyectos de inversión". Monografía. ED. CDICT- Facultad de Tecnología de la industria. Universidad Nacional de Ingeniería Managua, Nicaragua).

(Sánchez, I. R. (2005) "Evaluación financiera de proyectos de inversión”. Monografía. Universidad de Guayaquil de Ecuador).

(Coulter, M. Modern Chlor-Alkali Technology, 1980).

(Kelety Andrés. Análisis y evaluación de inversiones, 2da Edición, EADA, 1996).

(Kuznetsov D.Chemical Engeniering, Edición Mir. Moscú, 1969).

(Latiner, Wendel M and Hildebrand, Joel H. Reference book of Inorganic).

(Levenspiel, O. Chemical Reaction Engineering, 1972).

(Lewis, G. Industrial Chemistry. 1956).

(Lorie, J. Y Savage, L. Three Problems in Rationing Capital. In Foundation for Financial Management, Home Wood T 11: Irwin, 1966). 
(Mac Ketta, John and Cunningham, William. Encyclopedi of Chemical Processing and Desing, 1980).

(Menéndez Gutiérrez Carlos. Desechos peligrosos. MINSAP, 1997).

(Ministerio de Economía y Planificación. Metodología para la evaluación de los Estudios de Factibilidad de las Inversiones en Industrias. La Habana, 1976).

(Rodríguez Sandías, Alfonso. Análisis y Valoración de Proyectos. Universidad de Santiago. Departamento de Economía Financiera). 


\section{Para citar el artículo indexado.}

Oña B., Chacha H. Bravo A. Carrasco T. (2018). Indicadores económicos de las distribuidoras de maíz y su relación con la economía del cantón la maná. Revista electrónica Ciencia Digital 2(3), 50-60. Recuperado desde: http://cienciadigital.org/revistacienciadigital2/index.php/CienciaDigital/article/view/136/12 $\underline{1}$

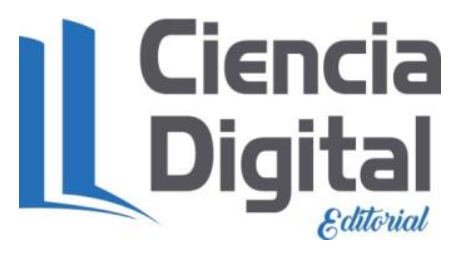

El artículo que se publica es de exclusiva responsabilidad de los autores y no necesariamente reflejan el pensamiento de la Revista Ciencia Digital.

El articulo queda en propiedad de la revista y, por tanto, su publicación parcial y/o total en otro medio tiene que ser autorizado por el director de la Revista Ciencia Digital.
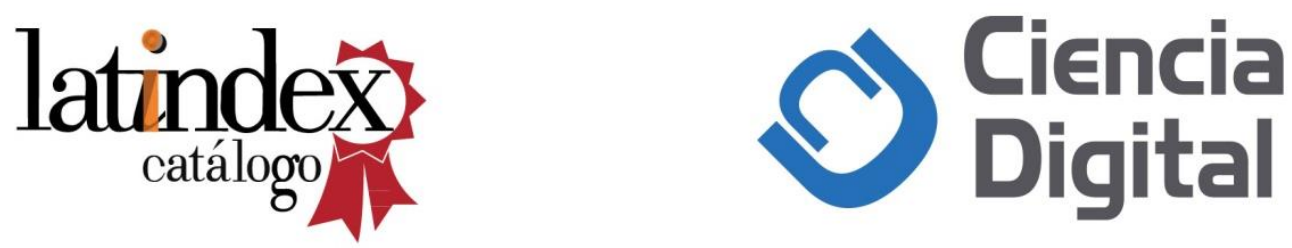